\title{
AN IMPROVED FUZZY CLUSTERING APPROACH FOR IMAGE SEGMENTATION
}

\author{
Ivana Despotović, Bart Goossens, Ewout Vansteenkiste and Wilfried Philips \\ Ghent Univesity, Dept. of Telecommunications and Information Processing, TELIN-IPI-IBBT, \\ St-Pietersnieuwstraat 41, B-9000 Ghent, Belgium
}

\begin{abstract}
Fuzzy clustering techniques have been widely used in automated image segmentation. However, since the standard fuzzy c-means (FCM) clustering algorithm does not consider any spatial information, it is highly sensitive to noise. In this paper, we present an extension of the FCM algorithm to overcome this drawback, by incorporating spatial neighborhood information into a new similarity measure. We consider that spatial information depends on the relative location and features of the neighboring pixels. The performance of the proposed algorithm is tested on synthetic and real images with different noise levels. Experimental quantitative and qualitative segmentation results show that the proposed method is effective, more robust to noise and preserves the homogeneity of the regions better than other FCM-based methods.
\end{abstract}

Index Terms - Image segmentation, Fuzzy clustering, Fuzzy C-Means, Spatial information

\section{INTRODUCTION}

Image segmentation plays a key role in image analysis and is often the first processing step in many image applications. The main goal of the image segmentation is to partition an image into a set of nonoverlapping, homogeneous regions with similar attributes such as intensity, depth, color, texture, etc. Since manual segmentation is timeconsuming and very often subjective and prone to errors, automated and accurate segmentation is needed. To date, various segmentation techniques have been developed and roughly, they can be grouped into five main categories: thresholding, edge-based methods, regionbased methods, neural network and clustering $[1,2]$. Since unsupervised fuzzy clustering is one of the most commonly used methods $[2,3]$ and has been successfully applied in fields such as astronomy, geology, medical and molecular imaging, it will be considered in this paper.

The main characteristic of fuzzy segmentation methods is to allow pixels to belong to multiple classes with certain degree, which is very useful in applications where uncertainty, limited spatial resolution and noise are present (for example satellite and medical images). Among fuzzy clustering methods, the fuzzy c-means (FCM) algorithm [4] is the most popular one. Since the conventional FCM algorithm classify pixels in the feature space without considering their spatial distribution in the image, it is highly sensitive to noise and other imaging artifacts. Many extensions of the FCM algorithm has been proposed [5-9] to overcome above mentioned problem and reduce errors in the segmentation process. The most common approach is to modify the FCM objective function $[5,6]$ or a similarity measure directly [7], by including spatial information. Ahmed et al. [5] modified the objective function of the standard FCM algorithm to allow the immediate neighbors of the pixel to influence its labeling. On the other hand, to keep the continuity from the FCM algorithm, Shen et al. [7] introduced a new similarity measure that depends on spatial neighborhood information, where the degree of the neighborhood attraction is optimized by a neural network. Beside those modifications, there are also other methods that can be used to enhance the FCM performance. For example, to improve the segmentation performance, one can combine the pixel-wise classification with pre-processing (noise cleaning in the original image) $[8,10]$ and post-processing (noise cleaning on the classified data). Xue et al. [10] proposed an algorithm where they firstly denoise images and then classify the pixels using the standard FCM method. All of these methods can reduce the noise to a certain extent, but still have some drawbacks such as increasing computational time [5], complexity $[5,7,9]$ and introducing unwanted smoothing $[8,10]$.

In this paper, we present an improved FCM clustering algorithm for image segmentation that integrates spatial neighborhood information into a similarity measure to overcome above mentioned problems. Spatial information depends on two neighborhood factors: the intensity similarity (feature attraction) and the relative spacial position (distance attraction) between the observed element and its neighboring elements. Experimental qualitative and quantitative results indicate that our method successfully reduces the effect of noise and biases the algorithm toward homogeneous clustering.

The paper is organized as follows. In Section 2, we explain the standard FCM method and our modified FCM algorithm. Experimental and comparison results are presented and discussed in Section 3. Finally, concluding remarks are given in Section 4.

\section{METHOD}

\subsection{FCM algorithm}

The FCM algorithm, initially developed by Dunn and later generalized by Bezdek [4], is an iterative, unsupervised, soft classification method. While hard classification methods (e.g. k-means) force pixels to belong exclusively to one class, FCM can retain much more information about the original image by allowing pixels to belong to multiple classes with different membership degrees.

Let $X=\left\{\mathbf{x}_{j}, j=1,2, \ldots, N \mid \mathbf{x}_{j} \in \mathbb{R}^{q}\right\}$ represent feature vectors of the image with $N$ pixels that needs to be partitioned into $C$ classes, where every component of the vector $\mathbf{x}_{j}$ represents a feature of the image at position $j$ and $q$ is the dimension of the feature vector. The FCM clustering algorithm is based on minimizing the following objective function:

$$
J_{m}=\sum_{i=1}^{C} \sum_{j=1}^{N} u_{i j}^{m} D_{i j},
$$

where $u_{i j}$ is the membership function of the feature $\mathbf{x}_{j}$ belonging to the $i$-th cluster, $m$ is the weighting exponent that controls the 
fuzziness of the resulting partition (most often is set to $m=2$ ) and $D_{i j}=d^{2}\left(\mathbf{x}_{j}, \mathbf{v}_{i}\right)$ is the similarity measure between $\mathbf{x}_{j}$ and the $i$-th cluster center $\mathbf{v}_{i}$. The most commonly used similarity measure is the squared Euclidean distance:

$$
D_{i j}=d^{2}\left(\mathbf{x}_{j}, \mathbf{v}_{i}\right)=\left\|\mathbf{x}_{j}-\mathbf{v}_{i}\right\|^{2} .
$$

The objective function $J_{m}$ (Eq. (1)) is minimized under the following constraints:

$$
u_{i j} \in[0,1], \sum_{i=1}^{C} u_{i j}=1 \forall j \text { and } 0<\sum_{j=1}^{N} u_{i j}<N \forall i,
$$

where low membership values are assigned to pixels far from the cluster centroid, and high membership values to pixels close to the cluster centroid. Considering the constraints $u_{i j}$ Eq. (3) and calculating the first derivatives of $J_{m}$ with respect to $u_{i j}$ and $\mathbf{v}_{i}$ and setting them to zero, results in two following conditions for minimizing $J_{m}$ :

$$
u_{i j}=\left[\sum_{k=1}^{C}\left(\frac{D_{i j}}{D_{k j}}\right)^{\frac{1}{m-1}}\right]^{-1}
$$

and

$$
\mathbf{v}_{i}=\frac{\sum_{j=1}^{N} u_{i j}^{m} \mathbf{x}_{j}}{\sum_{j=1}^{N} u_{i j}^{m}},(i=1,2, \ldots, C) .
$$

The FCM algorithm iteratively optimizes $J_{\mathrm{m}}$, by evaluating Eq. (4) and Eq. (5), until the following stop criterion is satisfied:

$$
\max _{i \in[1, C]}\left\|\mathbf{v}_{i}^{(l)}-\mathbf{v}_{i}^{(l+1)}\right\|_{\infty}<\epsilon,
$$

where $l$ is the iteration index and $\|\cdot\|_{\infty}$ is the $L_{\infty}$ norm. Once a membership value $u_{i j}$ for each class $i$ is assigned to each pixel $j$, a defuzzification of the fuzzy clusters $\left\{F_{k}\right\}_{k=1}^{C}$ into its crisp version $\left\{H_{k}\right\}_{k=1}^{C}$ is done by assigning the pixel to the class with the highest membership value as follows:

$$
\max _{i \in[1, C]}\left(u_{i j}\right)=u_{k j} \Longrightarrow \mathbf{x}_{j} \in H_{k} .
$$

The main drawback of the standard FCM for image segmentation is that the objective function does not take into account any spatial information and deals with the pixels as the separate points. Therefore, the standard FCM algorithm is sensitive to outliers and very often those pixels are wrongly classified.

\subsection{Proposed method}

Since the segmentation result in FCM algorithm is significantly influenced by membership values $u_{i j}$ (Eq. (1)) and considering the Eq. (4), we can conclude that the key to a successful segmentation is the choice of the similarity measure. Therefore, to improve the perofrmance of the FCM algorithm, we propose a new similarity measure as follows:

$$
D_{i j}=\left\|\mathbf{x}_{j}-\mathbf{v}_{i}\right\|^{2}\left(1-\alpha S_{i j}\right),
$$

where $S_{i j}$ represents the spatial neighborhood information and $\alpha \in$ $[0,1]$ is the parameter that controls the relative importance of the neighborhood attraction. If $\alpha=0, D_{i j}$ is the squared Euclidean distance and we have the standard FCM.

The spatial information $S_{i j}$ depends on the feature attraction $a_{j r}$ (pixel intensities) and the distance attraction $d_{j r}$ (relative location of neighboring pixels), and is defined as:

$$
S_{i j}=\frac{\sum_{r=1}^{N_{r}} u_{i r} a_{j r} d_{j r}^{-1}}{\sum_{r=1}^{N_{r}} a_{j r} d_{j r}^{-1}},
$$

where $N_{r}$ is the number of neighbors surrounding the element $\mathbf{x}_{j}$ in a square window $\Omega_{j}$ and $u_{i r}$ is the membership degree of the neighboring element $\mathbf{x}_{r}$ to the cluster $i$. If we define the neighborhood configuration $\Omega_{j}$ as an $n \times n$ square window with the center element $\mathbf{x}_{j}$, then $r=n^{2}-1$ and $\Omega_{j}=\left\{\mathbf{x}_{r} \mid r=1,2, \ldots, n^{2}-1\right\}$. Feature attraction $a_{j r}$ is defined as the absolute intensity differences between $\mathbf{x}_{j}$ and its neighbor $\mathbf{x}_{r}$

$$
a_{j r}=\left|\mathbf{x}_{j}-\mathbf{x}_{r}\right|
$$

The distance attraction $d_{j r}$ is the squared Euclidean distance between the coordinates of elements $\mathbf{x}\left(p_{j}, q_{j}\right)$ and $\mathbf{x}\left(p_{r}, q_{r}\right)$

$$
d_{j r}=\left(p_{j}-p_{r}\right)^{2}+\left(q_{j}-q_{r}\right)^{2} .
$$

In Eq. (9) we use the reciprocal of the distance $d_{j r}^{-1}$, because the neighbors $\mathbf{x}_{r}$ close to the center element $\mathbf{x}_{j}$ should more influence the result, while further neighbors should be less important. Fig. 1 illustrates the neighborhood configuration used in this work.

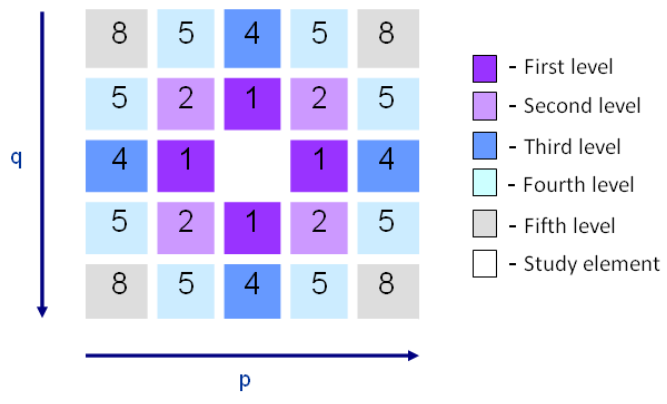

Fig. 1. Illustration of a neighborhood configuration (marked with colors and explained with the legend) and distance definition (squared Euclidean distance values are shown with numbers).

The idea behind this novel definition of spatial information is: Consider the local $n \times n$ neighborhood where the center element $\mathbf{x}_{j}$ has large intensity differences with the closest neighboring elements $\mathbf{x}_{r}$, which have similar intensities as the cluster center $\mathbf{v}_{i}$. If we calculate the neighborhood attraction $S_{i j}$, it will be large and the expression $\left(1-\alpha S_{i j}\right)$ will be small for $\alpha \neq 0$. After one iteration of the algorithm the central element $\mathbf{x}_{j}$ will be attracted to the cluster $i$. If the neighborhood attraction $S_{i j}$ is continuously large till the end of the algorithm, the central element $\mathbf{x}_{j}$ will be forced to belong to the cluster $i$ despite being dissimilar to it. Precisely, this property biases the algorithm towards homogeneous clustering.

The outline of the proposed algorithm is:

Step1. Set the number of clusters $C$, degree of fuzziness $m$, stop criterion $\epsilon$ and neighborhood size.

Step2. Initialize the centers of the clusters $\mathbf{v}_{i} \mid i=1,2, \ldots, C$.

Step3. Calculate the new similarity measure Eq. (8).

Step4. Calculate $u_{i j}$ using the new similarity measure Eq. (4).

Step5. Update $\mathbf{v}_{i}$ using $u_{i j}$ Eq. (5).

Repeat steps 3-5 until the stop criterion Eq.(6) is satisfied.

As with all clustering algorithms, the segmentation result may highly depend on the choice of parameter values used for initialization. Therefore, we use intensity-based thresholding [11] to reliably initialize the cluster centers. 


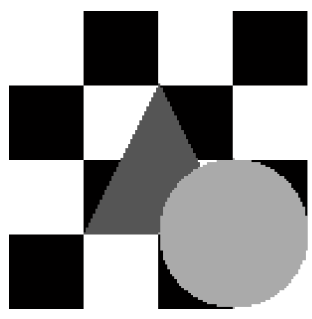

(a)

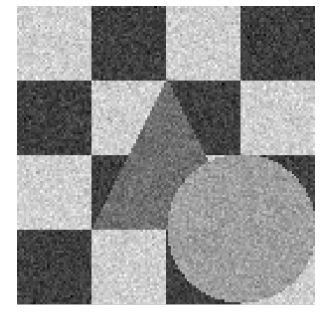

(b)

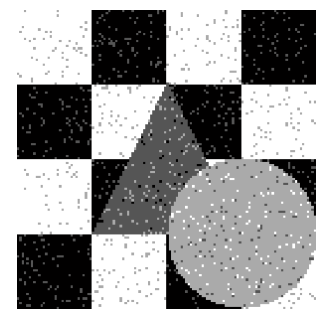

(c)

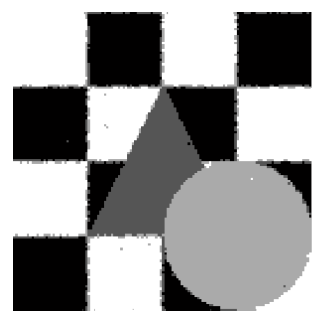

(d)

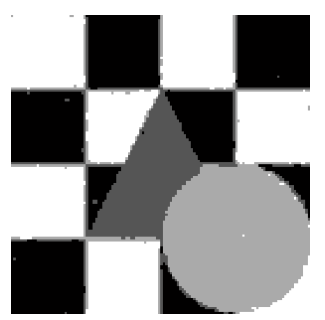

(e)

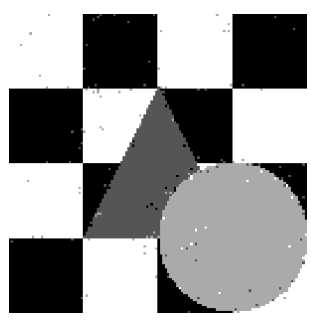

(f)

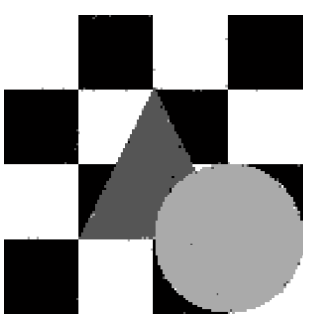

(g)

Fig. 2. Comparison of the segmentation results on a synthetic image with four gray levels and three different shapes: (a) original synthetic image; (b) the same image corrupted by zero mean Gaussian noise (SNR=16dB); (c) FCM [4]; (d) Ahmed et al. [5]; (e) Xue et al. [10]; (f) Shen et al. [7]; (g) our proposed algorithm.

\section{RESULTS}

In this section, the experimental results of our algorithm to synthetic and real images are presented. For all experiments we set the weighting exponent $m=2$, the stop criterion $\epsilon=0.01$, the neighborhood size $3 \times 3$ and the parameter which controls the effect of the neighbors $\alpha=1$.

To investigate the sensitivity of our proposed method to noise and to show the quantitative comparative results with other FCMbased methods $[4,5,7,10]$, we use the synthetic image (size $128 \times$ 128 ) shown in Fig. 2a. It contains four-class pattern with three different shapes and is corrupted by zero mean Gaussian noise (Fig. 2b), where Signal-to-Noise Ratio (SNR) between the original and noisy image is $16 \mathrm{~dB}$.

As can be seen in Fig. 2c, FCM algorithm can not classify correctly four classes, while results using Ahmed et al. [5] and Xue et al. [10] methods have edge blurring effects (Fig. 2d and e respectively). Shen et al. method succeed in classifying the data, but still with few remaining artefacts (Fig. 2f), while our proposed algorithm shows the best result (Fig. $2 \mathrm{~g}$ ).

In order to obtain a quantitative comparison, we plot the validation results of five methods for different noise levels in Fig. 3. The similarity index $\rho$, used for the comparison and quantitative evaluation, is the Dice coefficient:

$$
\rho=\frac{2\left|A_{i} \bigcap B_{i}\right|}{\left|A_{i}\right|+\left|B_{i}\right|},
$$

where $A_{i}$ and $B_{i}$ denote the set of pixels labelled into $i$ by the "ground truth" and our method respectively, and $\left|A_{i}\right|$ denotes the number of elements in $A_{i}$. In our experiment, the results for $\rho$ are averaged over all four classes.

From the Fig. 3 we can clearly see that our algorithm outperforms other FCM-based methods and acquires the best segmentation performance for all noise levels.

The performance of our algorithm is also demonstrated on real images, see Fig. 4 and Fig. 5.

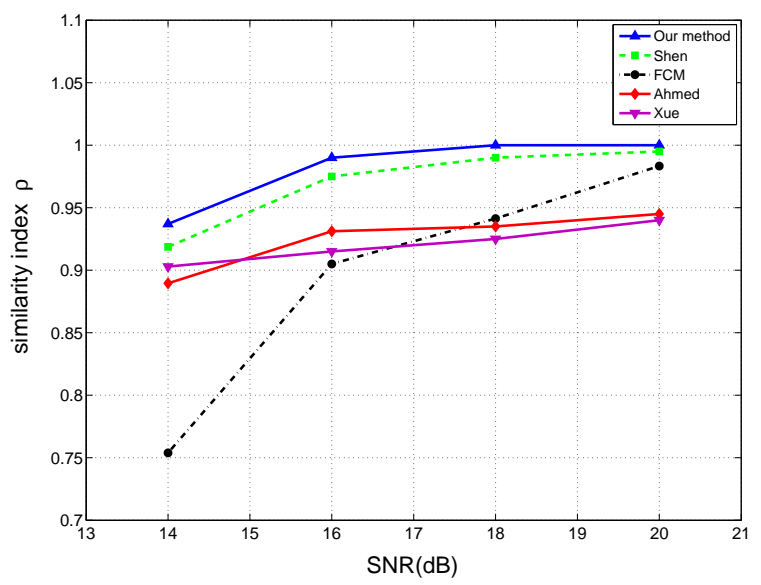

Fig. 3. Validation result for different noise levels. Comparison of FCM [4], Ahmed et al. [5], Xue et al. [10], Shen et al. [7] and our algorithm.

\section{CONCLUSION}

In this work, we have presented an improved FCM algorithm for unsupervised segmentation of noisy images. To enable robust segmentation and to overcome the disadvantages of the standard FCM algorithm, we integrated both, spatial and feature information of the image pixels into the segmentation algorithm. The quantitative and qualitative experimental results for simulated and real images show a good segmentation performance, especially for noisy images, and demonstrate an encouraging future of practical applications of the proposed method. 


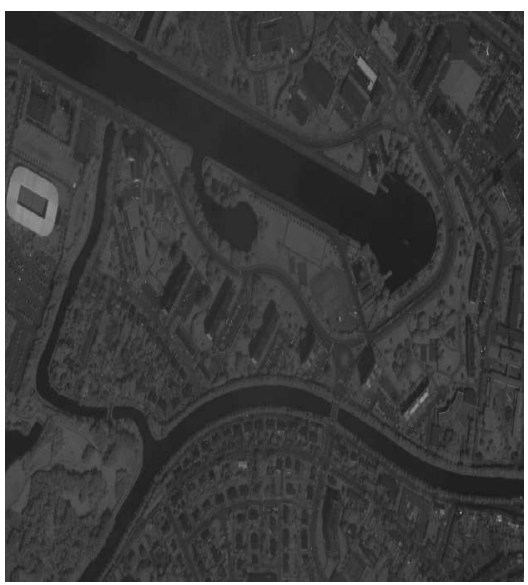

(a)

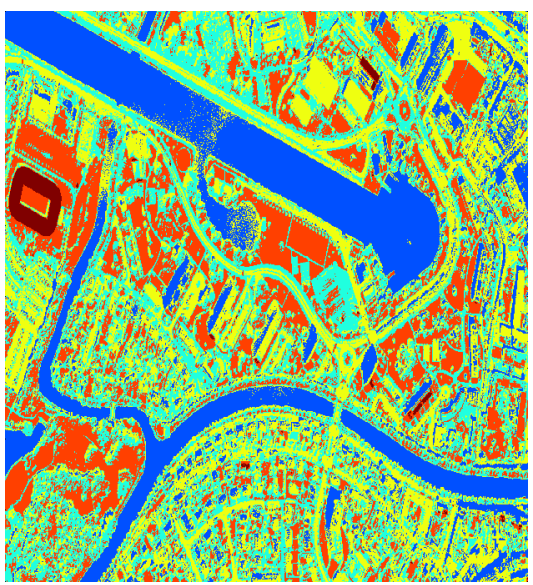

(b)

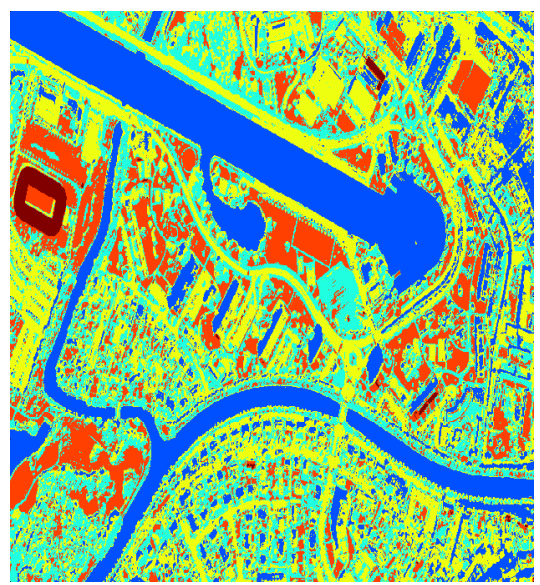

(c)

Fig. 4. Segmentation results on a satellite image: (a) original image; (b) FCM [4]; (c) our algorithm.
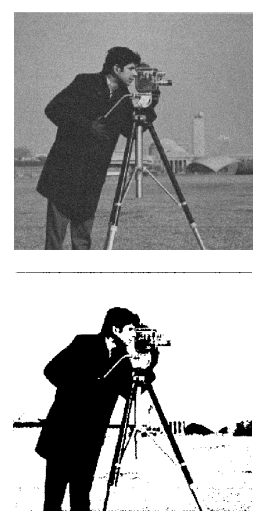

(a)
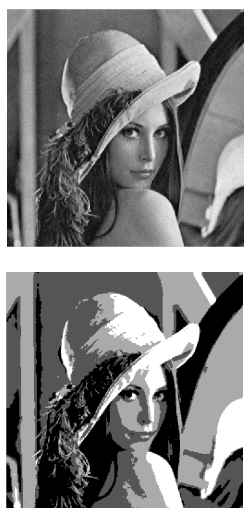

(b)
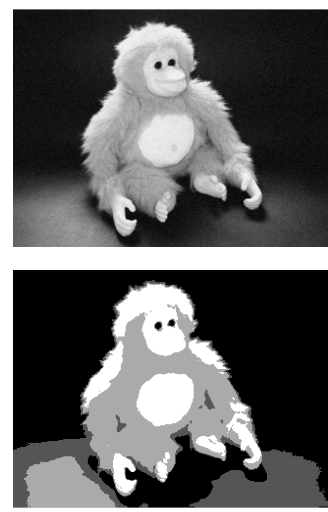

(c)
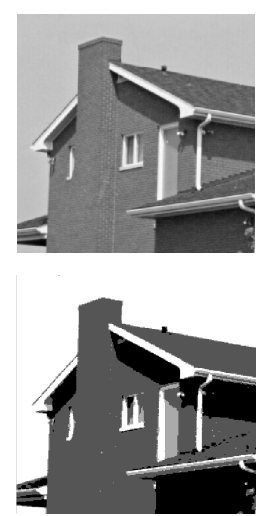

(d)
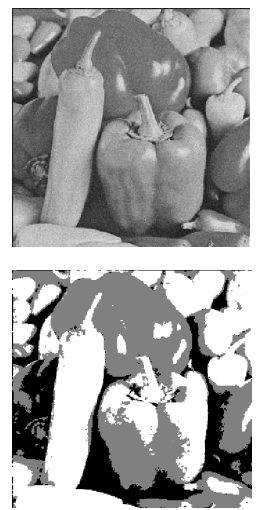

(e)
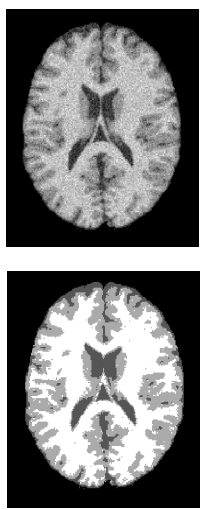

(f)

Fig. 5. Segmentation results on six real images. The first row shows the original images and the second row shows the results using our algorithm.

\section{References}

[1] R. Unnikrishnan, C. E. Pantofaru, and M. Hebert, "Toward objective evaluation of image segmentation algorithms," IEEE Trans. Pattern Anal. Machine Intell., vol. 29, pp. 929-943, 2007.

[2] N. Pal and S. Pal, "A review on image segmentation techniques," Pattern Recognition, vol. 26, pp. 1277-1294, 1993.

[3] J. V. de Oliveira and W. Pedrycz, Advances in Fuzzy Clustering and its Applications, John Wiley \& Sons, Inc., New York, NY, USA, 2007.

[4] J. C. Bezdek, Pattern Recognition with Fuzzy Objective Function Algorithms, Plenum Press, New York, 1981.

[5] M. N. Ahmed, S. M. Yamany, N. Mohamed, A. A. Farag, and T. Moriaty, "A modified fuzzy c-means algorithm for bias field estimation and segmentation of MRI data," IEEE Trans. Med. Imag., vol. 21, pp. 193-199, 2002.

[6] D. Zhang and C. Chen, "A novel kernelized fuzzy c-means algorithm with application in medical image segmentation," Artificial Inteligence in Medicine, vol. 32, pp. 37-50, 2004.
[7] S. Shen, W. Sandham, M. Granat, and A. Sterr, "MRI fuzzy segmentation of brain tissue using neighborhood attraction with neural-network optimization," IEEE Trans. Inf. Technology in Biomedicine, vol. 9, pp. 459-467, 2005.

[8] W. Cai, S. Chen, and D. Zang, "Fast and robust fuzzy c-means clustering algorithms incorporating local information for image segmentation," Pattern Recognition, vol. 40, pp. 825-838, 2007.

[9] Z. M. Wang, Y. C. Soh, Q. Song, and K. Sim, "Adaptive spatial information-theoretic clustering for image segmentation," Pattern Recognition Letters, vol. 42, pp. 2029-2044, 2009.

[10] J.-H. Xue, A. Pižurica, W. Philips, E. Kerre, R. Van de Walle, and I. Lemahieu, "An integrated method of adaptive enhancement for unsupervised segmentation of MRI brain images," Pattern Recognition Letters, vol. 24, pp. 2549-2560, 2003.

[11] S. S. Reddi, S. F. Rudin, and H. R. Keshavan, "An optical multiple threshold scheme for image segmentation," IEEE Trans. System Man Cybernet., vol. 14, pp. 661-665, 1984. 